\title{
Extracorporeal shockwave treatment impedes tooth movement in rats
}

\author{
Phimon Atsawasuwan ${ }^{1 *}$ (D), Yinghua Chen ${ }^{1}$, Karan Ganjawalla ${ }^{2}$, Albert L. Kelling ${ }^{3}$ and Carla A. Evans ${ }^{4}$
}

\begin{abstract}
Background: Accelerated tooth movement has been a topic of interest for orthodontic research recently. Surgically facilitated orthodontic treatment has been shown to be an effective approach to accelerate tooth movement; however, it remains invasive, requires additional surgery, and may increase post-operative complications. In this study, we evaluate the effects of extracorporeal shockwave treatment (ESWT), a non-invasive approach to regenerate alveolar bone, on orthodontic tooth movement in rats.

Materials and methods: Seventy-two male rats, aged 10 weeks old, were subjected to 10-cN closed-coil nickeltitanium springs for unilateral maxillary first molar tooth movement. One group of rats received a single treatment of extracorporeal shockwave treatment at 500 impulses at energy flux density $0.1 \mathrm{~mJ} / \mathrm{mm}^{2}$, with a pulse rate of 5 pulses per second immediately after spring installation while the non-ESWT-treated group served as a control group. The rats were sacrificed at day 3, 7, 14, 21 and 28 for tooth movement evaluation and sample analyses. Faxitron radiography, histological, double bone labeling and gene expression analyses were performed. Serum biochemistry was evaluated at day 3, 7 and 28 of the study. Kruskal-Wallis analysis of variance was used to determine the mean difference among groups, and multiple comparisons were analyzed by Mann-Whitney-U tests with a significance level $=0.05$.

Results: The results demonstrated that tooth movement in the ESWT-treated rats $(0.11 \pm 0.07 \mathrm{~mm})$ was impeded compared to the tooth movement in the non-ESWT-treated rats $(0.44 \pm 0.09 \mathrm{~mm})$. ESWT up-regulated several osteoblastic and osteoclastic gene markers and cytokines; however, the effects on osteoclasts were only transient. Double-fluorescence bone labeling demonstrated that osteoblastic activity increased after ESWT treatment. There was no difference in systemic RANKL/OPG ratio between groups.
\end{abstract}

Conclusions: ESWT at 500 impulse at energy flux density $0.1 \mathrm{~mJ} / \mathrm{mm}^{2}$ increased osteoblast and osteoclast activities and imbalanced bone remodeling resulting in impeded tooth movement in rats.

Keywords: Tooth movement, ESWT, Shockwave, Orthodontic, Rat

\section{Background}

Several approaches such as surgery, vibration/laser application, administration of biomolecules accelerate tooth movement; however, they demonstrate inconsistent results or provide insufficient evidence to reveal their effectiveness [1-9]. Increased alveolar bone traumatization is often used to maximize physiological healing cascades in a regional acceleratory phenomenon (RAP) with mineral release, localized osteoporosis (osteopenia), and increased osteoclast activities resulting in accelerated tooth movement [9]. However, the definite underlying

\footnotetext{
* Correspondence: patsawas@uic.edu

${ }^{1}$ Department of Orthodontics, University of Illinois at Chicago, College of

Dentistry, 801 S. Paulina St, Chicago, Illinois 60612, USA

Full list of author information is available at the end of the article
}

mechanism and extent of the effect is still controversial $[10,11]$. Despite the effectiveness of this approach, it remains invasive, requires additional surgery, and may increase post-operative complications.

Extracorporeal shockwave treatment (ESWT) is a non-invasive modality using a shockwave, an intense and very short energy wave traveling faster than the speed of sound [12]. ESWT has been used safely for augmentation of wound healing of bone and muscle and promotes alveolar bone regeneration, neovascularization, distraction osteogenesis and stem cell differentiation in rats [12-15]. ESWT has been used increasingly in healing of several refractory musculoskeletal disorders [1618] yet its biologic mechanism is not well understood

(c) The Author(s). 2018 Open Access This article is distributed under the terms of the Creative Commons Attribution 4.0 International License (http://creativecommons.org/licenses/by/4.0/), which permits unrestricted use, distribution, and 
$[12,13]$. Recently ESWT has been reported to induce interleukin-1 $\beta$ (IL-1 $\beta$ ), a potent cytokine for bone resorption, during 3 days of tooth movement in rats and no long-term observation was studied [19]. However, a study in humans failed to show any accelerated effect on second mandibular mesialization [20] after a single treatment of ESWT. Though ESWT promotes healing and induces tissue regeneration, the effect on tooth movement is controversial with different parameters /applications and tested species and subjects [19,20]. This study evaluated whether ESWT affects tooth movement in a rat model to gain better insight into underlying mechanisms.

\section{Methods}

\section{Tooth movement and ESWT application}

Seventy-two male Sprague-Dawley rats, aged 10 weeks (300-350 g; Charles River Laboratories, Wilmington, MA) were used in this study carried out in accordance with the recommendations in the Guide for the Care and Use of Laboratory Animals of the National Institutes of Health. The protocol was approved by the animal care committee, the University of Illinois at Chicago (ACC 12-183). During the course of the study, the rats were fed ad libitum with powdered rat chow and kept at a 12-h light cycle in the animal facility of the University of Illinois at Chicago. After anesthetized with ketamine and xylazine, all rats were subjected to 10-cN closed-coil springs (American Orthodontics, Sheboygan, WI) to mesialize their right maxillary first molars (ortho) [21] while the left maxillary first molars were left untouched (Fig. 1a) and served as the no orthodontic force (no ortho) control. Stainless steel ligature was used to ligate the spring between the first maxillary molar and the maxillary incisors then self-etching primer $(3 \mathrm{M}$ Unitek, Maplewood, MN) was applied over the ligations followed by light-cure flowable composite resin (3 M Unitek) to secure the wire and ensure stability. The rats were randomly divided into 2 groups: non-shockwave-treated controls (no ESWT; $n=38$ ) and shockwave treated group (ESWT; $n=34$ ). In the ESWT group, a single shockwave treatment of focused 500 impulses at energy flux density (EFD) $0.1 \mathrm{~mJ} / \mathrm{mm}^{2}$, with a pulse rate of 5 pulses per second was applied on the edentulous area on each side of the maxillae (Fig. 1b) [22]. A shockwave device (Vetgold ${ }^{\circ}$, Tissue Regeneration Technology LLC, Atlanta, GA) was used to deliver shockwaves on the rats' maxillae. Double-fluorescence bone labeling by intraperitoneal injection of calcein $(10 \mathrm{mg} / \mathrm{kg})$ was performed on day 0 and 24 and with alizarin-red $(30 \mathrm{mg} / \mathrm{kg})$ on day 14 .

\section{Faxitron radiography and microcomputed tomogram $(\mu \mathrm{CT})$ imaging}

Faxitron radiographic imaging (Faxitron X-ray Corp, Wheeling, IL) was performed at $3 \mathrm{X}$ magnification with

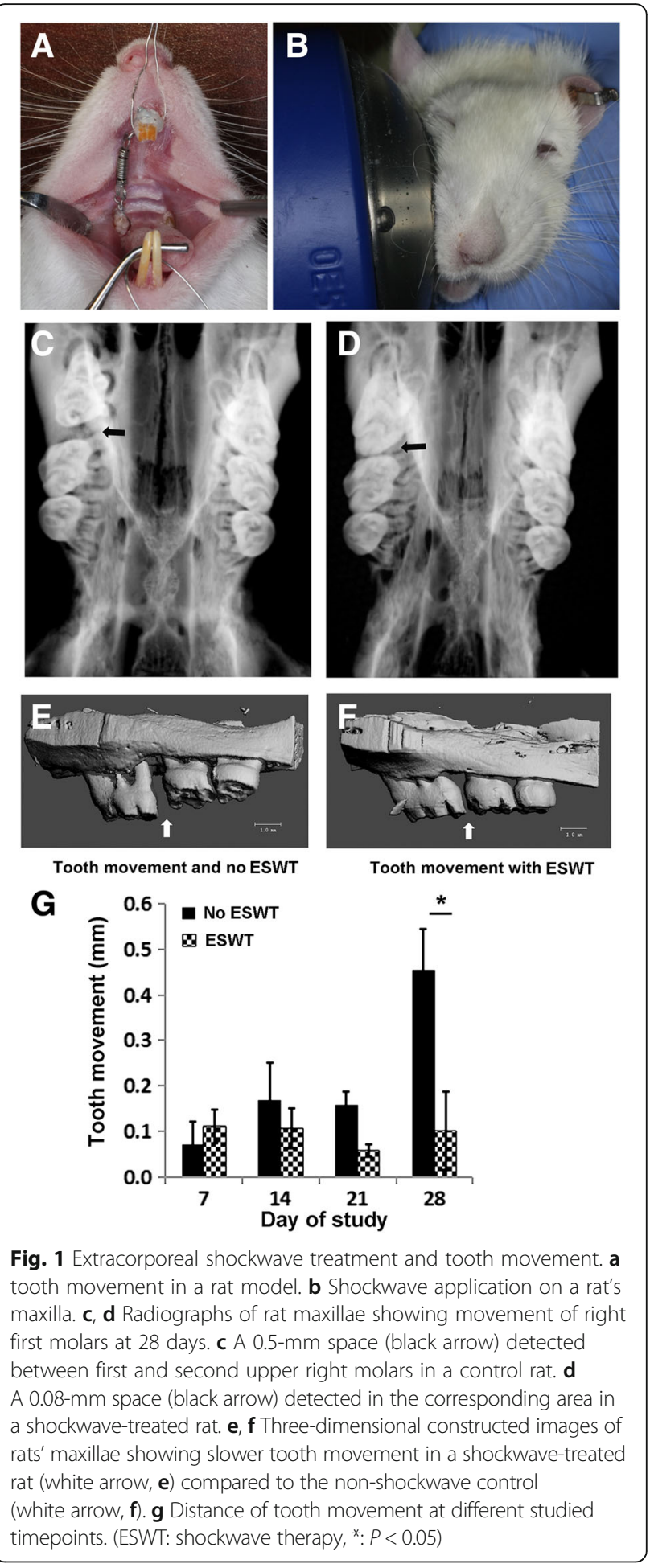

$35 \mathrm{kV}$ for $45 \mathrm{~s}$ for all samples. Mesial molar movement was determined by comparing the sagittal distance of the molar position on each side relative to the rats' second molar. The tooth movement measurement was performed with a ruler tool in Adobe photoshop program (Adobe Systems, San Jose, CA). Hemimaxillae were then scanned with Scanco 
$\mu$ CT40 (Scanco Medical, Switzerland) at a parameter of $70 \mathrm{kV}, 114 \mu \mathrm{A}, 300 \mathrm{~ms}$ and $10 \mu \mathrm{m}$ voxel size to render 3-dimensional $\mu \mathrm{CT}$ images and bone parameter analysis.

\section{Gene expression study}

At day 7, 14 and 28 of the study, alveolar bone segments mesial to (compression) and distal to (tension) the first maxillary molars were dissected under a stereomicroscope. The corresponding bone samples on the contralateral sides were collected as the no orthodontic force (no ortho) controls. The bone samples were pulverized under liquid nitrogen and kept in TRiZol at $-80^{\circ}$ until use. Total RNA extraction was performed with RNeasy kit (Qiagen, Carlsbad, CA), subjected to a nanodrop spectrophotometer to verify the quality of RNA. cDNA was generated with Omniscript RT kit (Qiagen) following the manufacturer's instructions. To quantify mRNA expression levels, realtime-PCR was performed using sequence-specific Sybergreen primers (Table 1) and normalized using $\beta$-actin. The analyses were performed using Quanstudio 3 Real-Time PCR system (Thermo Fisher Scientific, Waltham, MA). Relative expression levels were calculated using the $2^{-} \Delta \Delta^{\mathrm{Ct}}$ method [23].

\section{Histology}

Hematoxylin and eosin (H\&E) and tartrate-resistant acid phosphatase (TRAP) (Sigma-Aldrich, St Louis, MO) stainings were performed at day 28. The area of interest was delineated as $400 \mu \mathrm{m}^{2}$ area of interradicular bone of the first maxillary molar and located $200 \mu \mathrm{m}$ from the mesiobuccal root of the first maxillary molar. For fluorescent microscopic imaging, the non-demineralized hemimaxillae at day 28 were processed in methyl methacrylate and cut using a grinder (Exakt Technologies, Oklahoma City, OK). The sections were evaluated using ImageJ program under a fluorescent microscope (EVOS FL, Life Technologies, Grand Island, NY).

\section{Serum biochemistry}

On days 3, 7 and 28 of the study, the blood/serum from each animal was collected, processed and subject to osteoprotegerin (OPG) (Mybiosource, San Diego, CA) and receptor activator of nuclear factor kappa-B ligand (RANKL) (R\&Dsystems, Minneapolis, MN) ELISA kits and the ratio was calculated.

\section{Statistical analysis}

The experiments were performed in triplicate. Kruskal-Wallis analysis of variance was used to determine the mean difference among groups, and multiple comparisons were analyzed by Mann-Whitney-U tests with a significance level $=0.05$.

\section{Results}

\section{Distance of tooth movement}

All rats were viable and showed no sign of lethargy after spring installation. Faxitron radiographs demonstrated a smaller distance between first and second molars in shockwave-treated rats $(0.11 \pm 0.07 \mathrm{~mm})$ compared to the distance in the controls $(0.44 \pm 0.09 \mathrm{~mm})$ (Fig. 1c, d) on day 28 of study. The $\mu \mathrm{CT}$ confirmed delayed tooth movement in shockwave-treated rats (Fig. 1e, f) but the significant difference between groups was found on day $28(P<0.05)$ (Fig. 1g).

\section{Gene expression study}

On day 7, increased expression of osteoclast markers: cathepsinK (CtsK), acid phosphatase (Acp), and osteoblast markers: collagen I (Col1), osteocalcin (Ocn) was observed in both compression and tension samples of the shockwave-treated rats, compared to the corresponding samples from the no ESWT (mesial and distal) controls (Fig. 2a). With the absence of orthodontic force, a 10-25-fold increase of all studied genes was observed on distal control sites of ESWT group compared to the corresponding site of no ESWT controls $(P<0.05)$ while a 5-10-fold increase was observed on mesial control sites of ESWT group compared to the no ESWT controls $(P<0.05)$. In the presence of orthodontic force, ESWT did not exhibit any positive effect on osteoclast markers and did exhibit only a $2-5$-fold positive effect on osteoblast markers $(P<0.05)$. On day 14 , with the

Table 1 Primer sequences for realtime-PCR

\begin{tabular}{lll}
\hline Name/Accession number & Forward primer & Reverse primer \\
\hline Tracp-5b & 5'CAGCTTCCACCCTGAGATTC3' & 5'CGGTTCTGGCGATTTCTTTA3' \\
NM_001270889.1 & 5'GACCAGCGAAGAAGTGGTTC3' & 5'GACTCTGCCTTCCCACTCTG3' \\
CathepsinK & & \\
NM_031560.2 & 5'AAGACCTCCCGCCTGCCCAT3' & 5'CACGAAGCAGGCAGGGCCAA3' \\
Col1a1 & 5'TGAGGACCCTCTCTCTGCTC3' & 5'TGGACATGAAGGCTTTGTCA3' \\
NM_053304.1 & Ssteocalcin & 5'AGCCATGTACGTAGCCATCC3' \\
NM_013414.1 & & 5'ACCTCATAGATGGGCACAG3' \\
$\beta$ actin & & \\
\hline NM_031144.3
\end{tabular}




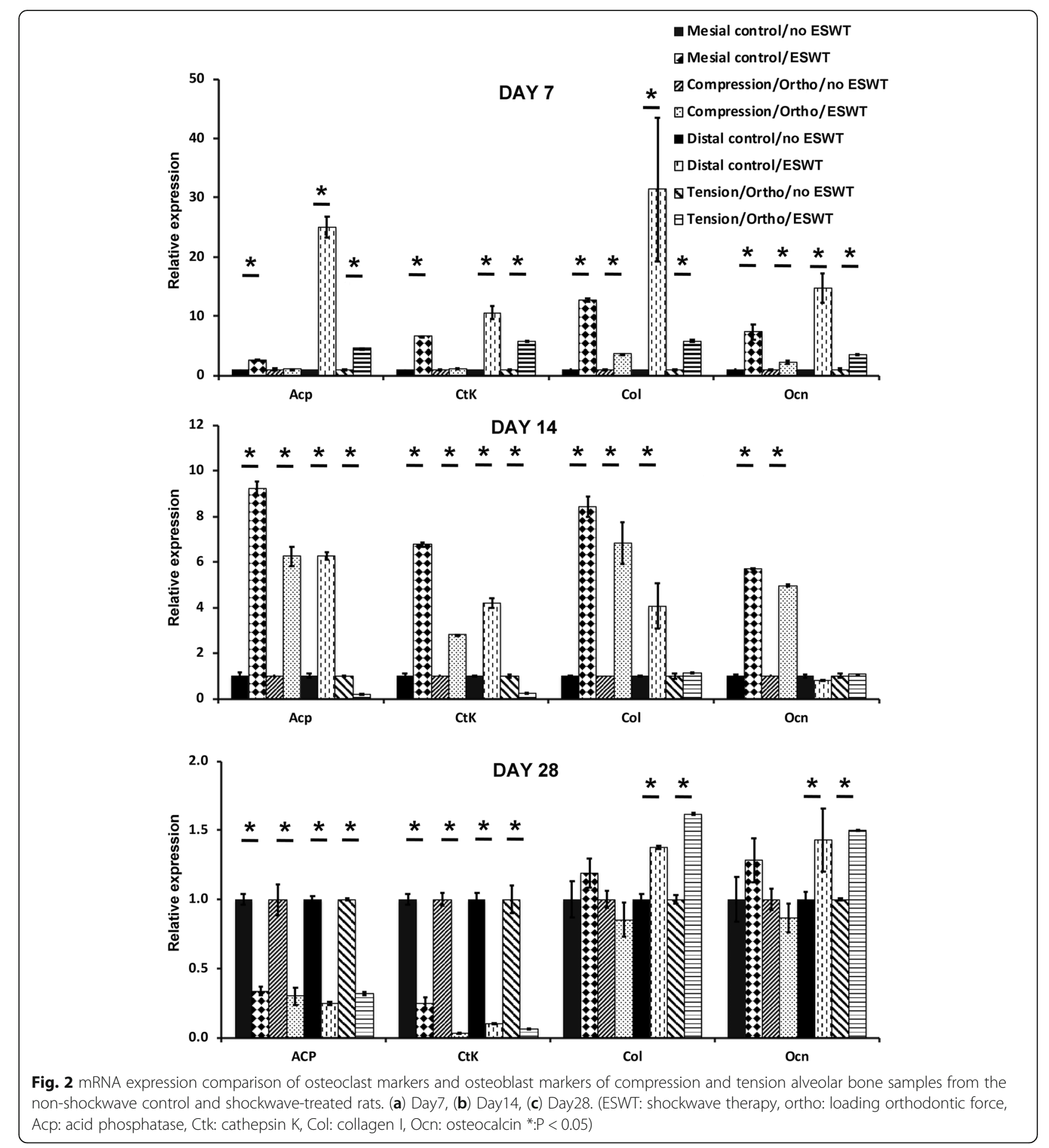

absence of orthodontic force, a 5-10-fold increase expression of osteoclast and osteoblast markers was observed on the compression areas of ESWT groups compared to no ESWT groups $(\mathrm{P}<0.05)$ (Fig. $2 \mathrm{~b})$. In the presence of orthodontic force, a 2-6-fold increase of osteoclast markers and osteoblast markers was observed on the compression side but a 3-8-fold decrease of osteoclast markers was observed on the tension side $(P<0.05)$. On day 28, the expression of all osteoclast markers decreased significantly in all groups. The positive effects of ESWT on osteoblast markers were observed on day 28 but a significant increase was found only in the tension sides of ESWT groups. These demonstrated that the positive effects of ESWT on osteoblasts lasted until the end of study $(\mathrm{P}<0.05)$ (Fig. 2c) while ESWT exhibited negative effects on osteoclast at the end of study $(P<0.05)$ (Fig. 2c). 


\section{Histological studies}

At day 28, in the presence of orthodontic force, both no ESWT (Fig. 3b) and ESWT (Fig. 3d) groups exhibited decreased periodontal ligament space width on the compression side but wide space on the tension side. Increased numbers of osteoclasts were observed at interradicular bone lacunae on the compression side and increased interradicular bone resorption was detected in both no ESWT and ESWT with orthodontic force (Fig. 3b, d) compared to those of no orthodontic force groups (Fig. 3a, c). Relative higher bone volume was detected in the ESWT group (Fig. 3c, d) compared to the no ESWT groups (Fig. 3a, b). TRAP staining (Fig. 3e) was performed to identify the osteoclasts at day 14 and 28 . The result revealed significantly increased number of osteoclasts after a single treatment of shockwave treatment (ESWT/no ortho) when compared to the non-shockwave-treated and no orthodontic force control group (no ESWT/no ortho) (Fig. 3f). However, the increased number of osteoclasts was significant only on day 14 , (Fig. 3f; $P<0.05$ ) not on day 28 (Fig. 3f; $P>0.05$ ). In the presence of orthodontic force, increased numbers of osteoclasts were detected in both no ESWT and ESWT groups (Fig. 3f; P < 0.05); however, there was no difference between both groups at any timepoint. In contrast, in the absence of orthodontic force, increased numbers of osteoclasts were detected only at day 14 of ESWT group (Fig. 3f; P > 0.05). The result may imply that the positive effects of ESWT on osteoclast numbers were only transient and synergistic in the presence of orthodontic force. However, in the presence of
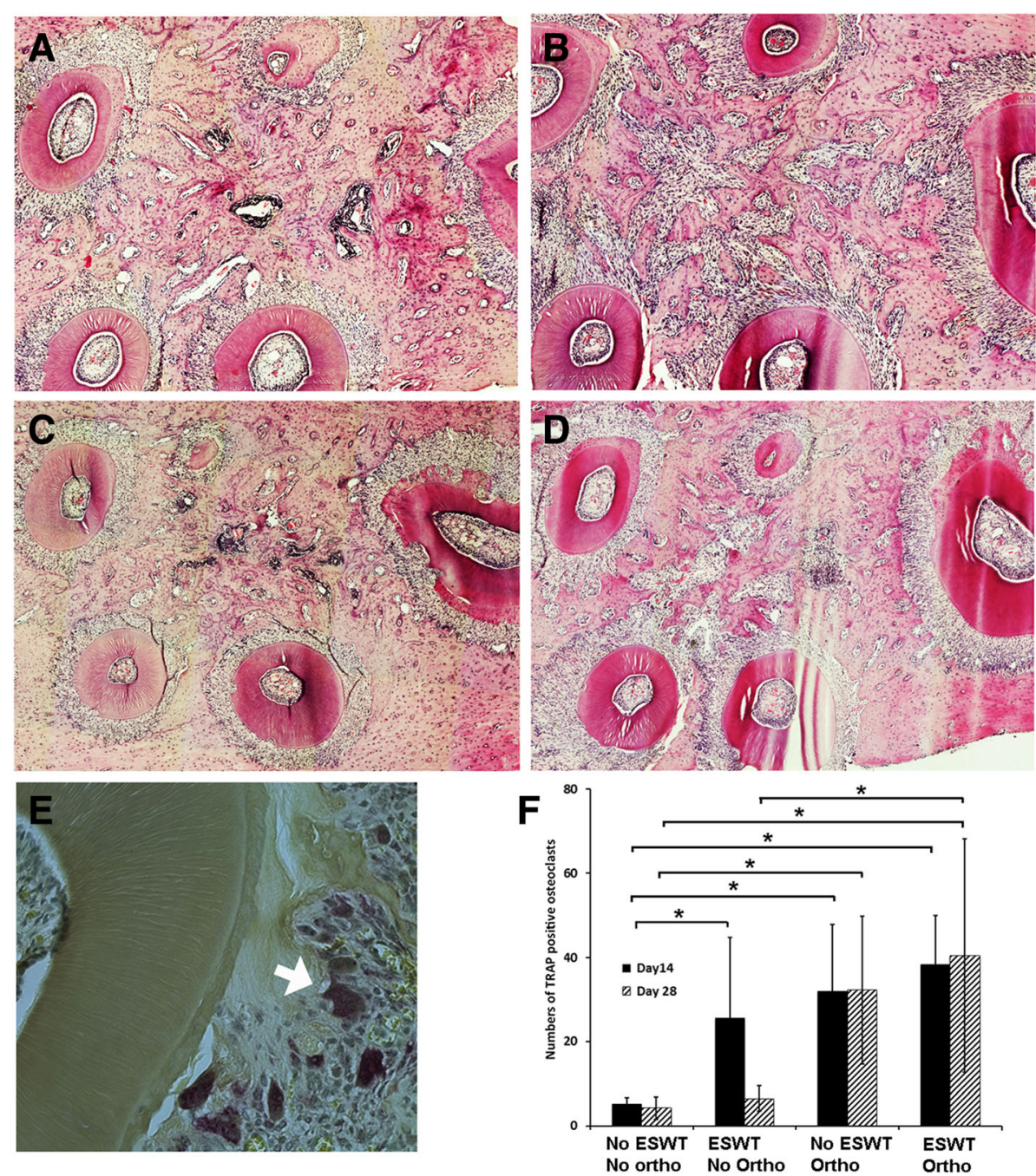

Fig. 3 Histological sections of orthodontic-treated and/or shockwave-treated rats' maxillae. $\mathbf{a}, \mathbf{b}, \mathbf{c}$, $\mathbf{d}$ H\&E sections of interradicular alveolar bone of a maxillary first molar from each group (a) no shockwave-treated and no orthodontic force control (no ESWT/no ortho), (b) no shockwavetreated with orthodontic force group (no ESWT/ortho), (c) shockwave-treated without orthodontic-treated group (ESWT/no ortho), (d) shockwavetreated with orthodontic-treated group (ESWT/ortho). e High magnification view of TRAP positive osteoclasts (arrow). $\mathbf{f}$ The number of osteoclasts of the studied areas of each studied group at day 14 and $28(*: P<0.05)$ 
orthodontic force, the effect of ESWT on osteoclasts was not striking.

\section{Double bone labeling assay and serum biochemistry}

At day 28, non-demineralized maxillae sections elucidated more prominent fluorescence signals from calcein and alizarin Red S in the ESWT groups (Fig. 4c, d) compared to the no ESWT groups (Fig. 4a, b), indicating increased bone apposition activity in the ESWT groups (Fig 4e). The orthodontic treatment alone increased bone remodeling activity compared to the non-orthodontic treatment control (Fig. 4a, e). ESWT alone (Fig. 4c, e) showed increased bone apposition as increased fluorescence signal compared to the non-orthodontic and non-shockwave control (Fig. 4a, e). The combination of shockwave treatment and orthodontic treatment showed the most prominent fluorescence signals among all samples (Fig. 4d, e). The ratio of serum RANKL and OPG from both ESWT and no ESWT rats was calculated at day 3, 7 and 28. No significant differences were found between both groups at any time-points indicating that the effect of shockwaves was localized and did not affect systemic activity of osteoclasts (Fig. 4f).

\section{Discussion}

This study is the first to demonstrate that a single treatment of ESWT impedes tooth movement in rats, affects osteoclast differentiation and increases osteoblast activity. Increased levels of cytokines and osteoblast/clast markers play an important role in recruitment of osteoclasts and osteoblasts leading to active bone remodeling during tooth movement $[24,25]$. Several previous studies showed that ESWT affects inflammatory and angiogenic cytokine expression and osteoblast activities with subsequent bone regeneration [26-30]. In this study, ESWT led to increased osteogenic activity and osteoblast marker expression similar to previous reports [19, 31]. The effects of ESWT were clearly observed when ESWT was used without orthodontic force. The positive effects of ESWT on osteoblasts lasted until the end of the study while the positive effects on osteoclast markers and numbers were transient. This phenomenon indicated imbalanced activities of osteoblast and osteoclast after ESWT treatment; however, the net positive effects of ESWT was on osteoblasts. In this study, though the focused applicator was used to limit the area of shockwave
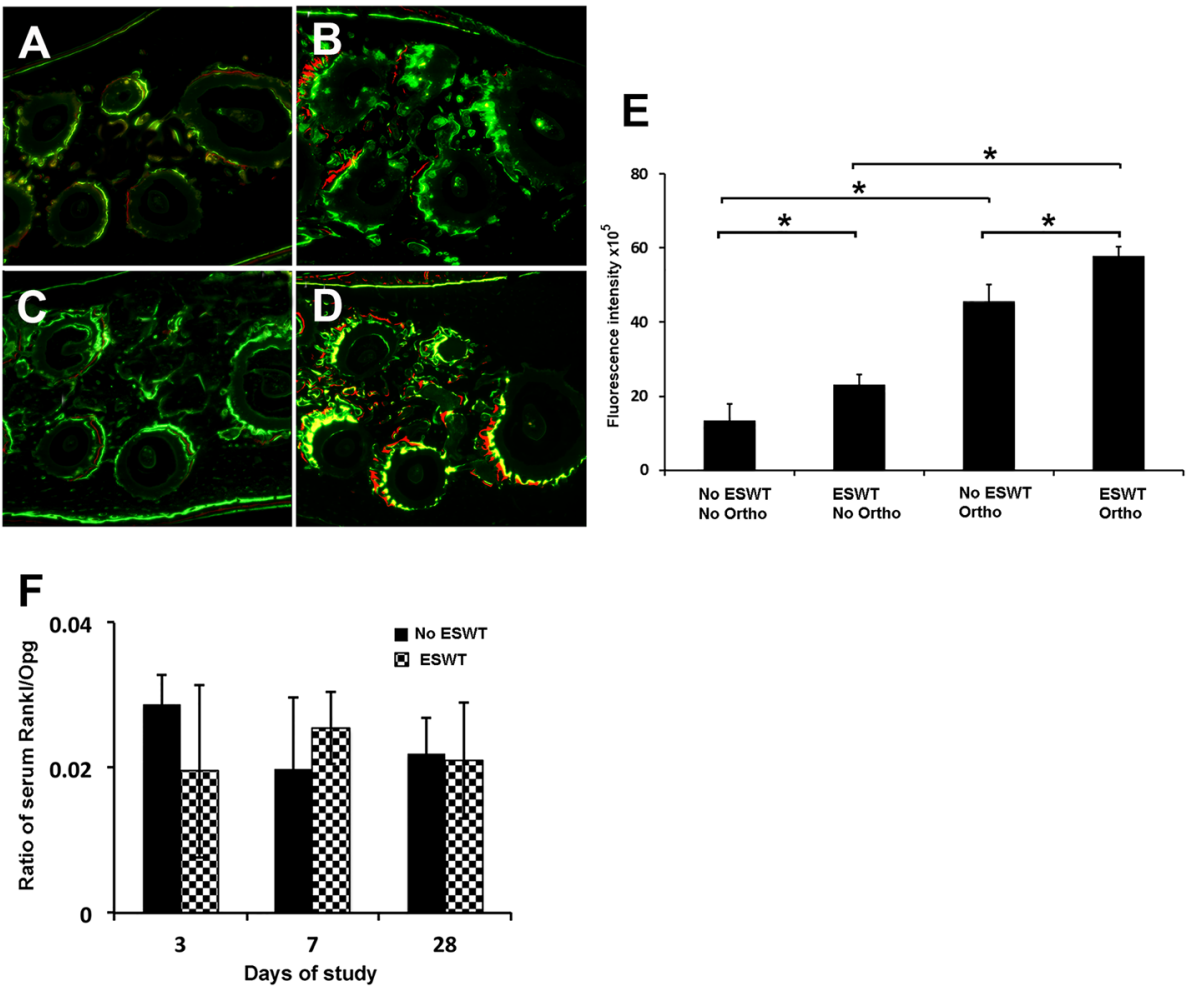

Fig. 4 Double-fluorescence bone labeling with calcein (green color) and alizarin (red color) signal on cross sections of maxillae from 4 groups viewed under a fluorescent microscopy $(\mathbf{a}, \mathbf{b}, \mathbf{c}, \mathbf{d})$. a no shockwave treatment and no orthodontic treatment control (no ESWT/no ortho), (b) no shockwave treatment with orthodontic treatment group (no ESWT/ortho), (c) shockwave treatment without orthodontic treatment group (ESWT/ no ortho), (d) shockwave treatment with orthodontic treatment (ESWT/ortho). Increased fluorescent signal was detected in shockwave-treated groups both orthodontic-treated (c) and non-orthodontic-treated groups $\mathbf{d}$ ). e Comparison of fluorescence intensity of sections from each group. f The serum RANKL/OPG ratio of both shockwave- and non-shockwave groups at day 3, 7 and 28 of the study. No significant difference was found at any timepoints indicating no systemic effect after a single application of shockwave at early or late timepoint 
treatment, the effect on the surrounding tissues is difficult to eliminate. We evaluated the systemic effect of ESWT on other surrounding areas using ratio of serum OPG and RANKL to determine systemic activity of osteoclasts [32] during the study. No difference of the ratio of serum OPG and RANKL was found between ESWT and control groups indicating no systemic effect of ESWT after a single application. The effects of ESWT application seemed to be local. Several studies reported that ESWT increased bone mineral content and bone mineral density in osteoporotic patients [31, 33]. In our study, H\&E and double bone labeling demonstrated increased bone apposition and osteoblast markers expression of ESWT rats' maxilla sections, which implicated the positive effects of ESWT on osteoblasts. Several studies demonstrated that ESWT promoted osteogenesis in human bone [34-36] and increased several growth factors for healing [36]. We speculate that the reduced tooth movement after a single treatment of ESWT was due to imbalanced activities of osteoblasts and osteoclasts during tooth movement.

\section{Limitations}

Only one specific set of parameters of ESWT was used in the study. The effects of shockwave could be varied due to different parameters, including intensity and frequency of the shockwaves as well as the timing of application of shockwaves in relation to when tooth movement is started. The relapse animal model could be used to evaluate the effect of ESWT on relapse as well.

\section{Conclusions}

1. A single application of ESWT of focused 500 impulses at EFD $0.1 \mathrm{~mJ} / \mathrm{mm}^{2}$, with a pulse rate of 5 pulses per second applied at the time tooth movement was initiated impeded tooth movement in rats.

2. A single application of ESWT in this study promoted both osteoblastic and osteoclastic activities, however, there were net positive effects exhibited on osteoblasts along with imbalance of the bone remodeling process. ESWT had no systemic effect on osteoclast activity.

\begin{abstract}
Abbreviations
Acp: Acid phosphatase; Col1: Collagen l; CtsK: Cathepsin K; EFD: Energy flux density; ESWT: Extracorporeal shockwave therapy; H\&E: Hematoxylin and eosin; IL-1ß: Interleukin-1beta; Ocn: Osteocalcin; OPG: Osteoprotegerin; RANKL: Receptor activator of nuclear factor kappa-B ligand; RAP: Rapid acceleratory phenomenon; TRAP: Tartrate-resistant acid phosphatase
\end{abstract}

\section{Acknowledgements}

We thank Tissue Regeneration Technologies, LLC for loaning the shockwave device.

\section{Funding}

This work was supported by ROAAP fund, the University of Illinois at Chicago, Brodie Craniofacial Endowment fund and Biomedical Research Award, the American Association of Orthodontists Foundation and DE024531 the National Institute of Dental and Craniofacial Research, National Institute of Health.

\section{Availability of data and materials}

The datasets generated during and/or analysed during the current study are available from the corresponding author on reasonable request.

\section{Authors' contributions}

PA/YC/KG conducted experiments, and data interpretation and analysis. PA ALK and CAE designed the experiments. PA/ALK/CAE helped writing the manuscript. All of the authors provided the feedback on the preparation of manuscript. All authors read and approved the final manuscript.

\section{Ethics approval}

The study was carried out in accordance with the recommendations in the Guide for the Care and Use of Laboratory Animals of the National Institutes of Health. The protocol was approved by the animal care committee, the University of Illinois at Chicago (ACC 12-183).

\section{Competing interests}

AK hold Patent \# 7,118,540 which is related to the subject of this study. Other authors declare no potential conflicts of interest with respect to the authorship and/or publication of this article.

\section{Publisher's Note}

Springer Nature remains neutral with regard to jurisdictional claims in published maps and institutional affiliations.

\section{Author details}

${ }^{1}$ Department of Orthodontics, University of Illinois at Chicago, College of Dentistry, 801 S. Paulina St, Chicago, Illinois 60612, USA. ${ }^{2}$ Harvard School of Dental Medicine, Boston, Massachusetts, USA. ${ }^{3}$ Raleigh, USA. ${ }^{4}$ Department of Orthodontic and Dentofacial Orthopedics, Boston University, Boston, Massachusetts, USA.

Received: 28 March 2018 Accepted: 24 October 2018

Published online: 12 November 2018

\section{References}

1. Hashimoto F, Kobayashi Y, Mataki S, Kobayashi K, Kato Y, Sakai H. Administration of osteocalcin accelerates orthodontic tooth movement induced by a closed coil spring in rats. Eur J Orthod. 2001;23:535-45.

2. Collins MK, Sinclair PM. The local use of vitamin $D$ to increase the rate of orthodontic tooth movement. Amer J Orthod Dentofac Orthop. 1988;94: 278-84.

3. Brooks PJ, Heckler AF, Wei K, Gong SG. M-CSF accelerates orthodontic tooth movement by targeting preosteoclasts in mice. Angle Orthod. 2011;81:277-83.

4. Soma S, Matsumoto S, Hiquchi Y, Takano-Yamamoto T, Yamashita K, Kurisu K, et al. Local and chronic application of PTH accelerates tooth movement in rats. J Dent Res. 2000;79:1717-24.

5. Darendeliler MA, Zea A, Shen G, Zoellner H. Effects of pulsed electromagnetic field vibration on tooth movement induced by magnetic and mechanical forces: a preliminary study. Aust Dent J. 2007;52:282-7.

6. Nishimura M, Chiba M, Ohashi T, Sato M, Shimizu Y, lgarashi K, et al. Periodontal tissue activation by vibration: intermittent stimulation by resonance vibration accelerates experimental tooth movement in rats. Amer J Orthod Dentofac Orthop. 2008:133:572-83.

7. Kau CH, Kantarci A, Shaughnessy T, Vachiramon A, Santiwong P, de la Fuente $\mathrm{A}$, et al. Photobiomodulation accelerates orthodontic alignment in the early phase of treatment. Prog Orthod. 2013;14:30.

8. Yamaguchi M, Hayashi M, Fujita S, Yoshida T, Utsunomiya T, Yamamoto $\mathrm{H}$, et al. Low-energy laser irradiation facilitates the velocity of tooth movement and the expressions of matrix metalloproteinase-9, cathepsin $\mathrm{K}$, and alpha(v) beta(3) integrin in rats. Eur J Orthod. 2010;32:131-9.

9. Alikhani M, Raptis M, Zoldan B, Sangsuwon C, Lee YB, Alyami B, et al. Effect of micro-osteoperforations on the rate of tooth movement. Amer J Orthod Dentofac Orthop. 2013;144:639-48.

10. Baloul SS, Gerstenfeld LC, Morgan EF, Carvalho RS, Van Dyke TE, Kantarci A. Mechanism of action and morphologic changes in the alveolar bone in response to selective alveolar decortication-facilitated tooth movement Amer J Orthod Dentofac Orthop. 2011;139:583-101.

11. Teixeira CC, Khoo E, Tran J, Chartres I, Liu Y, Thant LM, et al. Cytokine expression and accelerated tooth movement. J Dent Res. 2010;89:1135-41. 
12. Huang F, Kuo HK, Hsieh CH, Wu PC, Wu YC, Wang CJ. Effect of extracorporeal shockwave treatment on the melanogenic activity of cultured melanocytes. Appl Biochem Biotech. 2012;166:632-9.

13. Trebinjac S, Mujic-Skikic E, Ninkovic M, Karaikovic E. Extracorporeal shock wave therapy in orthopaedic diseases. Bosnian J Basic Med. 2005;5:27-32.

14. Lai JP, Wang FS, Hung CM, Wang CJ, Huang CJ, Kuo YR. Extracorporeal shock wave accelerates consolidation in distraction osteogenesis of the rat mandible. J Trauma. 2010;69:1252-8.

15. Mackert GA, Schulte M, Hirche C, Kotsougiani D, Vogelpohl J, Hoener B, Fiebig T, Kirschner S, Brockmann MA, Lehnhardt M, Kneser U, Harhaus L. Low-energy extracorporeal shockwave therapy (ESWT) improves metaphyseal fracture healing in an osteoporotic rat model. PLoS One. 2017; 12(12):e0189356

16. Wolfl C, Schuster L, Honer B, Englert S, Klein R, Hirche C, Munzberg M, Grutzner PA, Kneser U, Harhaus L. Influence of extracorporeal shock wave therapy (ESWT) on bone turnover markets in organisms with normal and low bone mineral density during fracture healing: a randomized clinical trial. GMS Interdiscip Plast Reconstr Surg DGPW. 2017;18:6 Doc 17.

17. Yu X, Zhang D, Chen X, Yang J, Shi L, Pang Q. Effectiveness of various hip preservation treatments for non-traumatic osteonecrosis of the femoral head: a network meta-analysis of randomized controlled trials. J Orthop Sci. 2017;17:30346-9

18. Vitali M, Naim Rodriguez N, Pedretti A, Drossinos A, Pironti P, Di Carlo G, Fraschini G. Bone marrow edema syndrom of the medial femoral condyle treated with extracorporeal shock wave therapy: a clinical and MRI retrospective comparative study. Arch Phys Med Rehabil. 2017;17:31392-8.

19. Hazan-Molina H, Reznick AZ, Kaufman H, Aizenbud D. Periodontal cytokines profile under orthodontic force and extracorporeal shock wave stimuli in a rat model. J Periodontal Res. 2015;50:389-96.

20. Falkensammer F, Arnhart C, Krall C, Schaden W, Freudenthaler J, Bantleon HP. Impact of extracorporeal shock wave therapy (ESWT) on orthodontic tooth movement-a randomized clinical trial. Clin Oral Investig. 2014;18: 2187-92.

21. Ren Y, Maltha JC, Kuijpers-Jagtman AM. The rat as a model for orthodontic tooth movement--a critical review and a proposed solution. Eur J Orthod. 2004:26:483-90.

22. Sathishkumar S, Meka A, Dawson D, House N, Schaden W, Novak MJ, et al. Extracorporeal shock wave therapy induces alveolar bone regeneration. J Dent Res. 2008;87:687-91.

23. Livak KJ, Schmittgen TD. Analysis of relative gene expression data using real-time quantitative PCR and the 2(-Delta Delta $C(T))$ method. Methods. 2001;25:402-8.

24. Ren Y, Vissink A. Cytokines in crevicular fluid and orthodontic tooth movement. Eur J Oral Sci. 2008;116:89-97.

25. Krishnan V, Davidovitch Z. On a path to unfolding the biological mechanisms of orthodontic tooth movement. J Dent Res. 2009;88:597-608.

26. Tam KF, Cheung WH, Lee KM, Qin L, Leung KS. Shockwave exerts osteogenic effect on osteoporotic bone in an ovariectomized goat model. Ultrasound Med Biol. 2009:35:1109-18.

27. Wang $\mathrm{CJ}$, Huang $\mathrm{CY}$, Hsu SL, Chen JH, Cheng JH. Extracorporeal shockwave therapy in osteoporotic osteoarthritis of the knee in rats: an experiment in animals. Arthritis Res Ther. 2014;16:R139.

28. Wang CJ, Wang FS, Yang KD. Biological effects of extracorporeal shockwave in bone healing: a study in rabbits. Arch Orthop Trauma Surg. 2008;128: 879-84.

29. Wang CJ, Yang KD, Ko JY, Huang CC, Huang HY, Wang FS. The effects of shockwave on bone healing and systemic concentrations of nitric oxide (NO), TGF-beta1, VEGF and BMP-2 in long bone non-unions. Nitric oxide-Biol Ch. 2009;20:298-303.

30. Martini L, Giavaresi G, Fini M, Borsari V, Torricelli P, Giardino R. Early effects of extracorporeal shock wave treatment on osteoblast-like cells: a comparative study between electromagnetic and electrohydraulic devices. J Trauma. 2006;61:1198-206.

31. Takahashi K, Yamazaki M, Saisu T, Nakajima A, Shimizu S, Mitsuhashi S, et al. Gene expression for extracellular matrix proteins in shockwave-induced osteogenesis in rats. Calcif Tissue Int. 2004;74:187-93.

32. Hofbauer LC, Kuhne CA, Viereck V. The OPG/RANKL/RANK system in metabolic bone diseases. J Musculoskelet Neuron Interact. 2004;4:268-75.

33. Shi L, Gao F, Sun W, Wang B, Guo W, Cheng L, Li Z, Wang W. Short-term effects of extracorporeal shock wave therapy on bone mineral density in postmenopausal osteoporotic patients. Osteopro Int. 2017;28:2945-53.
34. Gollwitzer H, Gloeck T, Roessner M, Langer R, Horn C, Gerdesmeyer L, Diehl P. Radial extracorporeal shock wave therapy (rESWT) induces new bone formation in vivo: results of an animal study in rabbits. Ultrashound med Biol. 2013:39:126-33.

35. Gerdesmeyer L, Schaden W, Besch L, Studenberg M, Doerner L, Muehlhofer $H$, Toepfer A. Osteogenetic effect of extracorporeal shock waves in human. Int J Surg. 2015;24:115-9.

36. Pfaff JA, Boelck B, Bloch W, Nentwig GH. Growth factors in bone marrow blood of the manddible with application of extracorporeal shock wave therapy. Implant Dent. 2016;35:179-86.
Ready to submit your research? Choose BMC and benefit from:

- fast, convenient online submission

- thorough peer review by experienced researchers in your field

- rapid publication on acceptance

- support for research data, including large and complex data types

- gold Open Access which fosters wider collaboration and increased citations

- maximum visibility for your research: over $100 \mathrm{M}$ website views per year

At BMC, research is always in progress.

Learn more biomedcentral.com/submissions 